\title{
A Ferroelastic Switching Model for Lead Zirconate-Titanate (PZT)
}

\author{
Brian L. Ball ${ }^{1}$, Ralph C. Smith ${ }^{2}$, Sang-Joo Kim ${ }^{3}$ and Stefan Seelecke ${ }^{4}$ \\ ${ }^{1,2}$ Center for Research in Scientific Computation \\ Department of Mathematics \\ North Carolina State University \\ Raleigh, NC 27695 \\ ${ }^{3}$ Mechanical and Information Engineering \\ University of Seoul \\ Seoul, 130-743 Korea \\ ${ }^{4}$ Mechanical and Aerospace Engineering \\ North Carolina State University \\ Box 7910 \\ Raleigh, NC 27695
}

\begin{abstract}
This paper develops a macroscopic polarization switching model which characterizes the ferroelastic switching mechanisms inherent to lead zirconate-titanate (PZT) in a manner suitable for subsequent transducer and control design. We construct Helmholtz and Gibbs energy relations at the lattice level which quantify the internal and electrostatic energy associated with $90^{\circ}$ and $180^{\circ}$ dipole orientations. Equilibrium relations appropriate for homogeneous materials in the absence or presence of thermal relaxation are respectively determined by minimizing the Gibbs energy or balancing the Gibbs and relative thermal energies using Boltzmann principles. Macroscopic models suitable for nonhomogeneous, polycrystalline compounds are constructed through stochastic homogenization techniques. Attributes and limitations of the model are illustrated through comparison with experimental PLZT data.
\end{abstract}

\section{Introduction}

This model, extending the work done in [1], combines energy principles at the lattice level, theory of thermally activated processes, and stochastic homogenization techniques to characterize hysteresis due to ferroelectric and ferroelastic switching in a manner which facilitates material characterization, transducer design, and model-based control design. Helmholtz and Gibbs energy relations are constructed at the lattice level to quantify the internal and electrostatic energy associated with $90^{\circ}$ and $180^{\circ}$ dipole orientations. For regimes in which thermal activation is significant, the Gibbs and relative thermal energies are balanced through Boltzmann theory to provide equilibrium relations quantifying local strains and polarizations as a function of input stresses and fields. In the limit of negligible

\footnotetext{
${ }^{1}$ Email: blball@unity.ncsu.edu, Telephone: (919) 332-7957

${ }^{2}$ Email: rsmith@eos.ncsu.edu, Telephone: (919) 515-7552

${ }^{3}$ Email: sjk@uos.ac.kr, Telephone: 82-2-2210-2757

${ }^{4}$ Email: stefan_seelecke@ncsu.edu, Telephone: (919) 515-5282
} 
thermal activation, the local relations reduce to minima of the Gibbs energy and enforcement of these minimization criteria significantly improves the efficiency of the model and inversion process employed for linear control design. Parameters such as the local coercive and interaction fields are considered to be manifestations of underlying distributions to incorporate the effects of polycrystallinity and material nonhomogeneities. Homogenization in this manner yields low-order models posed in terms of effective parameters that are efficient to implement in optimization or control algorithms.

Physical mechanisms associated with ferroelectric and ferroelastic switching are summarized in Section 2 to motivate $90^{\circ}$ and $180^{\circ}$ switching mechanisms that need to be incorporated in energy relations. In Section 3, we summarize the construction of a piecewise defined Gibbs energy functional. Minimization of this functional quantifies homogeneous, single crystal behavior in the absence of relaxation processes. In Section 4, stochastic homogenization techniques are employed to construct a macroscopic model for polycrystalline, nonhomogeneous materials and in Section 5, properties of the model are illustrated through comparison and prediction of experimental PLZT data.

\section{$2 \quad$ Ferroelectric and Ferroelastic Switching Mechanisms}

Lead zirconate-titanate is comprised of $\mathrm{PbZr}_{x-1} \mathrm{O}_{3}$ (lead zirconate) and $\mathrm{PbTi}_{x} \mathrm{O}_{3}$ (lead titanate) where $x$ is chosen to optimize electromechanical coupling. For temperatures above the Curie point $T_{c}$, the structures of $\mathrm{PbTiO}_{3}$ and $\mathrm{PbZrO}_{3}$ are cubic whereas for $T<T_{c}$, the structure of $\mathrm{PbTiO}_{3}$ is tetragonal and $\mathrm{PbZrO}_{3}$ is orthorhombic [6]. The switching mechanisms are illustrated in the context of the paraelectric cubic and the ferroelectric tetragonal structure of lead titanate and note the analogous behavior is observed for the orthorhombic structure of lead zirconate.

The application of an electric field $E$ that is larger in magnitude than the coercive field $E_{c}$ induces ferroelectric switching. For $\mathrm{PbTiO}_{3}$, this causes the central $\mathrm{Ti}^{+4}$ ion to relocate to a new equilibrium position, resulting in a $180^{\circ}$ change in polarization that is parallel to the applied field as depicted in Figure 1(a). Ferroelastic switching is caused by the application of a stress $\sigma$ that is larger in magnitude than the coercive stress $\sigma_{c}$ producing a $90^{\circ}$ change in polarization that is perpendicular to the applied stress as illustrated in Figure 1(b). The ferroelectric and ferroelastic switching mechanisms cause a hysteretic relationship between input fields $E$ and $\sigma$ and output polarization $P$ and strains $\varepsilon$ as illustrated in Figure 2.

At point A in Figures 2(a) and 2(b), the electric field is sufficiently strong so that all the dipoles form one domain that is aligned in the direction of the applied field. As the field is decreased, it approaches the negative coercive field in the region around point $\mathrm{B}$ where $180^{\circ}$ switching commences. Additionally, this often includes $90^{\circ}$ switching as indicated by the presence of a negative strain at point $\mathrm{B}$ as depicted in Figure 2(b). At point $\mathrm{C}$, all the dipoles have switched and again form one domain that is aligned in the direction of the electric field. At point $\mathrm{C}$, the polarization is opposite to that at point $\mathrm{A}$ whereas the strains have the same value. As the field is increased, $90^{\circ}$ switching occurs at point $\mathrm{D}$ and rapidly

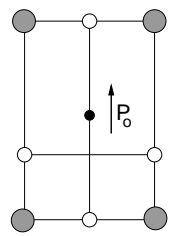

(a)

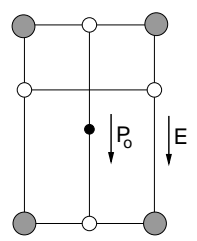

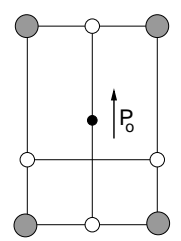

(b)

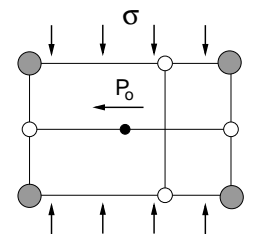

Figure 1: (a) Ferroelectric $180^{\circ}$ switch in spontaneous polarization $P_{0}$ induced by an applied electric field, and (b) ferroelastic $90^{\circ}$ switch induced by an applied stress. 


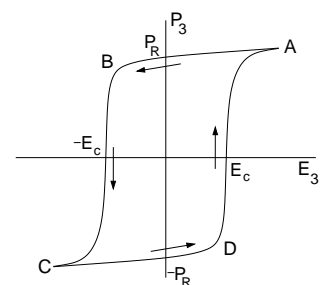

(a)

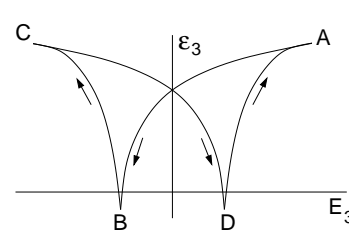

(b)

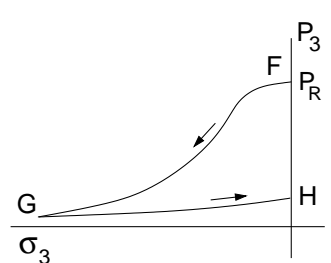

(c)

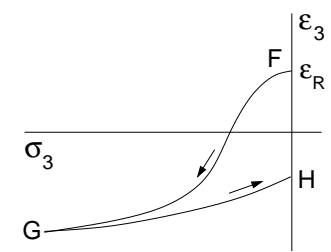

(d)

Figure 2: (a) Hysteretic field-polarization relation for bulk PLZT, (b) field-strain behavior of PLZT, (c) stress-polarization relation for PLZT, and (d) stress-strain behavior of PLZT.

continues back to point A where the full $180^{\circ}$ switch has occurred and the dipoles are again aligned with the applied electric field. Figures 2(c) and 2(d) illustrate the relationship between an externally applied stress and the polarization and strains. At point $\mathrm{F}$, the dipoles are aligned in the positive 3-direction and the material acts as one domain. As the compressive stress is increased in magnitude, it approaches the coercive stress. In the region around point $\mathrm{G}, 90^{\circ}$ switching occurs and the dipoles begin to align perpendicular to the direction of the applied stress. This is indicated by the presence of a negative strain at point $\mathrm{G}$ in Figure 2(d). As the stress is reduced in magnitude, the material remains poled perpendicular to the applied stress resulting in a decrease in the polarization in the 3-direction.

\section{$3 \quad$ 1-D Ferroelastic Switching Model}

To facilitate model implementation, improve computational efficiency and increase the model flexibility, we employ piecewise quadratic energy functionals which are approximations of energy functionals based on Landau-Devonshire principles. The model coefficients directly relate to measured material properties allowing a means for parameter identification and estimation. The low-order piecewise polynomial models also facilitate transducer and control design and incorporates both $90^{\circ}$ ferroelectric and ferroelastic polarization switching mechanisms. We note that aspects of the functionals are similar to those employed for SMA undergoing austinite - martinsite phase transformations and the reader is referred to $[3,6,7]$ for details illustrating properties of the SMA relations.

\subsection{Helmholtz and Gibbs Energy Relations}

We consider electric field and stress inputs $(E, \sigma)$ and polarization and strain outputs $(P, \varepsilon)$. To account for the $180^{\circ}$ and $90^{\circ}$ polarization switching, the polarization has three allowed dipole states $P_{-}, P_{+}$and $P_{90}$. We define the polarization component of the Helmholtz energy to be

$$
\psi_{p}(P)= \begin{cases}\frac{\eta}{2}\left(P+P_{R}\right)^{2} & , \quad P \leq-P_{I} \\ \frac{\eta_{1}}{2}\left(P+P_{m}\right)^{2}+\beta & , \quad-P_{I}<P<-P_{90 I} \\ \frac{\eta_{2}}{2}(P)^{2}+\Delta & , \quad|P| \leq P_{90 I} \\ \frac{\eta_{1}}{2}\left(P-P_{m}\right)^{2}+\beta & , \quad P_{90 I}<P<P_{I} \\ \frac{\eta}{2}\left(P-P_{R}\right)^{2} & , \quad P \geq P_{I}\end{cases}
$$


where

$$
\begin{array}{ll}
P_{m}=\frac{\eta\left(P_{I}-P_{R}\right) P_{90 I}-\eta_{2} P_{90 I} P_{I}}{\eta\left(P_{I}-P_{R}\right)-\eta_{2} P_{90 I}}, & \eta_{1}=\eta \frac{P_{I}-P_{R}}{P_{I}-P_{m}}, \\
\beta=\frac{\eta}{2}\left(P_{I}-P_{R}\right)^{2}-\frac{\eta_{1}}{2}\left(P_{I}-P_{m}\right)^{2}, & \Delta=\frac{\eta_{1}}{2}\left(P_{90 I}-P_{m}\right)^{2}+\beta-\frac{\eta_{2}}{2}\left(P_{90 I}\right)^{2} .
\end{array}
$$

The electromechanical coupling component is given by

$$
\psi_{e s}(P, \varepsilon)=-a \varepsilon P-q \varepsilon P^{2}
$$

where $a$ is the piezoelectric coupling coefficient and $q$ is the electrostrictive coupling coefficient. The elastic energy is

$$
\psi_{e l}(\varepsilon)=\frac{1}{2} Y \varepsilon^{2}
$$

where $Y$ is the Young's modulus. The total Helmholtz energy is then given by

$$
\psi(P, \varepsilon)=\psi_{p}(P)+\psi_{e s}(P, \varepsilon)+\psi_{e l}(\varepsilon) .
$$

Balancing the internal energy $\psi$ and the externally applied energy yields the Gibbs energy

$$
G(E, P, \sigma, \varepsilon)=\psi(P, \varepsilon)-E P-\sigma \varepsilon .
$$

The necessary condition $\frac{d G}{d \varepsilon}=0$ yields

$$
\varepsilon=Y^{-1}\left(\sigma+a P+q P^{2}\right) .
$$

In a manner analogous to that employed in [4] for a stress-free state, the strain is substituted into the Gibbs energy (3) resulting in a Gibbs energy of the form

$$
G(E, P, \sigma)=\widehat{\psi}(P, \sigma)-E P-\sigma \varepsilon \text {. }
$$

\subsection{Switching in the Absence of Thermal Activation}

Neglecting thermal effects, the local polarization $\langle P\rangle$ for a single crystal with an applied stress can be determined by solving the necessary condition $\frac{\partial G}{\partial P}=0$, or by minimizing the Gibbs energy

$$
\langle P\rangle=\arg \min _{P} G(E, P, \sigma)
$$

where the Gibbs energy is defined by equation (4).

To calculate the value of the coercive field, we apply the necessary condition $\frac{\partial G}{\partial P}=0$ and solve for the value of the electric field that results in $P=P_{I}$ to obtain

$$
E_{c}(\sigma)=\frac{2 q P_{I}-a}{Y}(\sigma)+\frac{2 q^{2} P_{I}^{3}}{Y}-\frac{3 a q P_{I}^{2}}{Y}+\frac{a^{2} P_{I}}{Y}+\eta\left(P_{R}-P_{I}\right) .
$$

It is clear from (6) that the coercive field is stress dependent. The coercive stress $\sigma_{c}$ can be computed by setting $E_{c}=0$ in (6). This results in

$$
\sigma_{c}=\frac{1}{a-2 q P_{I}}\left[\eta Y\left(P_{R}-P_{I}\right)+2 q^{2}\left(P_{I}\right)^{3}-3 a q\left(P_{I}\right)^{2}+a^{2}\left(P_{I}\right)\right] .
$$

The electric field required to eliminate the $90^{\circ}$ minima in the Gibbs energy is denoted by $E_{c}^{90}$. An explicit expression for the value of $E_{c}^{90}$ is found to be

$$
E_{c}^{90}(\sigma)=-(\sigma) \frac{a+2 q P_{90}}{Y}-\frac{2 q^{2} P_{90}^{3}+3 a q P_{90}^{2}+\left(a^{2}-\eta_{2} Y\right) P_{90}}{Y} .
$$

A direct $180^{\circ}$ ferroelectric polarization switch (i.e., $P_{ \pm} \rightarrow P_{\mp}$ ) can occur if $E_{c}^{90}<E_{c}$. A $90^{\circ}$ ferroelectric polarization switch (i.e., $P_{ \pm} \rightarrow P_{90}$ ) can occur if $E_{c}^{90}>E_{c}$ and a $90^{\circ}$ ferroelastic polarization switch will occur when $\sigma>\sigma_{c}$. 


\subsection{Switching in the Presence of Thermal Activation}

For the switching criteria summarized in Section 3.2, dipole switching occurred only when the magnitude of the applied electric field or stress exceeded that of the coercive field or stress value of the single crystal. However, mechanisms such as excitation from thermal effects can induce switching before local minima are eliminated. To model this phenomena, we incorporate the effects of thermal activation. We continue to assume that the material is homogeneous and that each dipole in this collection has the same energy landscape and are subject to the same switching mechanisms. This allows us to model the switching that occurs by evolving the fraction of dipoles in each allowed orientation by a population model that is similar to what is derived in [8].

In the manner detailed in [6], thermal activation processes are incorporated through the use of the Boltzmann relation

$$
\mu(G(P))=C e^{-G(P) V / k T} .
$$

Here $V$ denotes a representative control volume, $k$ is Boltzmann's constant and $T$ is the temperature. The fraction of dipoles in each allowed orientation is given by $x_{-}, x_{90}$ and $x_{+}$. Conservation of the number of dipoles yields

$$
x_{-}+x_{90}+x_{+}=1
$$

The local polarization $\langle P\rangle$ is given by

$$
\langle P\rangle=x_{-}\left\langle P_{-}\right\rangle+x_{90}\left\langle P_{90}\right\rangle+x_{+}\left\langle P_{+}\right\rangle
$$

where $\left\langle P_{-}\right\rangle,\left\langle P_{90}\right\rangle$ and $\left\langle P_{+}\right\rangle$are the expected polarization values associated with each allowed dipole orientation. As illustrated in $[6,8]$, these values are found by integrating the product of the polarization $P$ and the Boltzmann probability density $\mu(G(P))$ over the allowed polarization states. This simplifies to the relations

$$
\left\langle P_{-}\right\rangle=\frac{\int_{-\infty}^{-P_{I}} P e^{-\gamma G(P)} d P}{\int_{-\infty}^{-P_{I}} e^{-\gamma G(P)} d P}, \quad\left\langle P_{90}\right\rangle=\frac{\int_{-P_{90 I}}^{P_{90 I}} P e^{-\gamma G(P)} d P}{\int_{-P_{90 I}}^{P_{90 I}} e^{-\gamma G(P)} d P}, \quad\left\langle P_{+}\right\rangle=\frac{\int_{P_{I}}^{\infty} P e^{-\gamma G(P)} d P}{\int_{P_{I}}^{\infty} e^{-\gamma G(P)} d P}
$$

where $\gamma=\frac{V}{k T}$. For computational efficiency, we can approximate these values by use of the necessary condition $\frac{\partial G}{\partial P}=0$ which yields

$$
\left\langle P_{-}\right\rangle=\frac{E}{\eta}-P_{R}, \quad\left\langle P_{90}\right\rangle=\frac{E}{\eta_{2}}, \quad\left\langle P_{+}\right\rangle=\frac{E}{\eta}+P_{R} .
$$

In this case, $\left\langle P_{-}\right\rangle,\left\langle P_{90}\right\rangle$ and $\left\langle P_{+}\right\rangle$are the location of the minima of the Gibbs energy.

The time evolution of the dipole phase fractions are governed by the first order ODE system

$$
\left(\begin{array}{c}
\dot{x_{-}} \\
x_{90} \\
x_{+}
\end{array}\right)=\left(\begin{array}{ccc}
-p_{-} & p_{90-} & 0 \\
p_{-} & -\left(p_{90-}+p_{90+}\right) & p_{+} \\
0 & p_{90+} & -p_{+}
\end{array}\right)\left(\begin{array}{c}
x_{-} \\
x_{90} \\
x_{+}
\end{array}\right)
$$

The system results from the assumption that transitions between the three allowed states occur only to the nearest neighbor and can be reduced in dimension by applying the conservation relation (10). The likelihood to switch out of the $P_{-}$orientation into the $P_{90}$ orientation is denoted by $p_{-}$and the notation for the remaining likelihoods is summarized in Table 1.

The likelihood $p_{-}$is calculated by

$$
p_{-}=\frac{1}{\tau} \frac{e^{-\gamma G\left(-P_{I}\right)}}{\int_{-\infty}^{-P_{I}} e^{-\gamma G(P)} d P}
$$




\begin{tabular}{c|c} 
State Transition & notation \\
\hline$P_{-} \rightarrow P_{90}$ & $p_{-}$ \\
$P_{90} \rightarrow P_{-}$ & $p_{90-}$ \\
$P_{90} \rightarrow P_{+}$ & $p_{90+}$ \\
$P_{+} \rightarrow P_{90}$ & $p_{+}$ \\
\hline
\end{tabular}

Table 1: 1-D transition likelihoods.

where the relaxation time $\tau$ is the reciprocal of the frequency at which dipoles attempt a switch. The likelihood of switching out of the $P_{90}$ orientation into the $P_{-}$orientation is specified by $p_{90}$ and is calculated by

$$
p_{90-}=\frac{1}{\tau} \frac{e^{-\gamma G\left(-P_{90}\right)}}{\int_{-P_{90}}^{P_{90}} e^{-\gamma G(P)} d P} .
$$

The likelihoods $p_{90+}$ and $p_{+}$are obtained in a similar manner. The likelihoods can also be evaluated in terms of error functions resulting in,

$$
p_{+}=\frac{1}{\tau} \frac{e^{-\gamma G\left(P_{I}\right)}}{\int_{P_{I}}^{\infty} e^{-\gamma G(P)} d P}=\frac{1}{\tau} \frac{e^{-\alpha\left(P_{I}+b / 2\right)^{2}}}{\frac{1}{2} \sqrt{\frac{\pi}{\alpha}} \cdot \operatorname{erfc}\left(\sqrt{\alpha}\left(P_{I}+b / 2\right)\right)}
$$

where $\alpha=\frac{\gamma \eta}{2}$ and $b=-2 \frac{\left(P_{R}-E\right)}{\eta}$. The likelihoods $p_{90+}, p_{90-}$ and $p_{-}$follow similarly.

\section{Macroscopic Polarization Model}

Nonuniformities in the lattice structure due to polycrystallinity, material nonhomogeneities and variations across grain boundaries produce a distribution of Helmholtz and Gibbs energy profiles which can be manifested as variations in the local coercive field and local remanent polarization. Other variations can be produced by stress nonhomogeneities and variable effective fields.

To incorporate these effects en route to constructing a macroscopic model, we consider the coercive field $E_{c}$ to be a manifestation of an underlying density $\nu_{1}\left(E_{c}\right)$ rather than fixed values which is typically assumed for single crystals having a uniform lattice structure. To create a macroscopic model for the polarization, we also consider the variation of effective fields in the material. As detailed in $[6,8]$, an applied field $E$ in a ferroelectric material is augmented by an interaction field $E_{I}$ generated by neighboring dipoles which produce nonhomogeneous effective fields in the material. This, along with various other processes, produces variations in the applied field that can significantly alter the resulting polarization. To incorporate these variations, we consider the effective field $E_{e}=E+E_{I}$ to be distributed about the applied field $E$ with an underlying density for the interaction field $E_{I}$ which we denote by $\nu_{2}\left(E_{I}\right)$. The introduction of variations in the effective field produces domain switching in advance of the remanence point in accordance with observations from experimental data.

The complete macroscopic polarization model for nonhomogeneous, polycrystalline materials with distributed coercive and effective fields is

$$
P(E)=\int_{0}^{\infty} \int_{-\infty}^{\infty}\langle P\rangle\left(E ; E_{I}, E_{c}\right) \nu_{1}\left(E_{c}\right) \nu_{2}\left(E_{I}\right) d E_{I} d E_{c}
$$

where $\langle P\rangle$ is the local polarization kernel given by (5) when thermal effects are negligible or by (11) when incorporating thermal relaxation. 


\section{$5 \quad$ Model Validation and Properties}

In this section, the ferroelastic model presented in Section 3 is compared with experimental PLZT data reported in [2]. The ferroelastic switching model employs the thermal activation model presented in Section 3.3 to evolve the polarization in time. However, since the measured values exhibit minimal relaxation, the switching model was computed with a large $\gamma$ and a small $\tau$ which resulted in negligable thermal activation. As reported in [2], the PLZT composition is also very near the rhombohedraltetragonal morphotropic boundary. To characterize this nonhomogenous composition, we utilize the macroscopic polarization model of Section 4.

For the macroscopic model, we make the a priori assumption that the density for the coercieve field is given by

$$
\nu_{1}\left(E_{c}(\sigma)\right)=\left\{\begin{array}{cc}
c_{1} e^{-\bar{E}_{c}^{2}(\sigma) / b_{1}} & e^{-\bar{E}_{c}^{2}(\sigma) / b_{1}}>0 \\
0 & e^{-\bar{E}_{c}^{2}(\sigma) / b_{1}} \leq 0
\end{array}\right.
$$

and the density for the interaction field is given by

$$
\nu_{2}\left(E_{I}\right)=c_{2} e^{-E_{I}^{2} / b_{2}} .
$$

The relation $\bar{E}_{c}(\sigma)$ is specified by the single crystal value (6) and the variance of the coercive field was estimated from the variance in the experimentally measured values in Table 2 . The variance of the effective field was estimated by the degree to which switching occurs before remanence in the PLZT data. The parameters of the densities $\nu_{1}\left(E_{c}\right)$ and $\nu_{2}\left(E_{I}\right)$ used in the model were $c_{1}=27.0$, $b_{1}=6.5 \times 10^{-4}(\mathrm{MV} / \mathrm{m})^{2}, c_{2}=6.0$ and $b_{2}=5.0 \times 10^{-3}(\mathrm{MV} / \mathrm{m})^{2}$. To implement the macroscopic polarization given by (12), a composite Gaussian quadrature was employed.

The slope of the hysteron in the $180^{\circ}$ regime is given by $\eta^{-1}$, the slope of the hysteron in the $90^{\circ}$ regime is given by $\eta_{2}^{-1}$ and the remanent polarization is defined by the parameter $P_{R}$. The remaining coefficients $a, q$ and $Y$ affect the slope and intercept of the linear $E_{c}(\sigma)$ relation $(6)$ and these values can be ascertained by fitting the $E_{c}(\sigma)$ relation to experimental data given in Table 2 . The values of $P_{m}, \eta_{1}, \beta$ and $\Delta$ are defined in Equation (1). The value of the parameters used to model the PLZT data are compiled in Table 3.

The model is employed with varying applied stresses as well as an oscillating electric field to match experimental conditions; the reader is referred to [2] for details regarding the experimental procedures. The ferroelastic model is compared to the PLZT data in Figures $3-5$. The model parameters were chosen to optimize the fit of the $E-P$ and $E-\varepsilon$ data shown in Figures 3 and 4 . The ferroelastic model characterizes the $90^{\circ}$ switching that occurs in the $E-P$ data as a compressive stress is applied to the PLZT sample. The model also predicts a negative strain due to an applied compressive stress as well as the butterfly nature of the $E-\varepsilon$ data.

It is noted that a possible source of error in the model fit may arise from the rhombohedral nature of the PLZT data since the ferroelastic model is derived in the tetragonal phase.

The datafit of the $\sigma-\varepsilon$ relation shown in Figure 5 can be optimized by setting the Young's modulus parameters to match the slope of the appropriate part of the $\sigma-\varepsilon$ curve. However, this results in an

\begin{tabular}{c|c|c|c}
$\sigma(M P a)$ & $P_{R}\left(C / m^{2}\right)$ & $E_{c}(M V / m)$ & $E_{c}^{90}(M V / m)$ \\
\hline 0 & 0.247 & $0.35 \pm 0.01$ & $0.35 \pm 0.01$ \\
-6 & 0.247 & $0.26 \pm 0.01$ & $0.35 \pm 0.01$ \\
-10 & 0.235 & $0.19 \pm 0.02$ & $0.35 \pm 0.02$ \\
-15 & 0.215 & $0.15 \pm 0.02$ & $0.39 \pm 0.02$ \\
\hline
\end{tabular}

Table 2: Experimental values for $\sigma, P_{R}, E_{c}^{90}$, and $E_{c}$ for rhombohedral PLZT from data reported in [2]. 


\begin{tabular}{c|c|c|c} 
parameter & value & parameter & value \\
\hline$P_{R}$ & $0.24\left(\mathrm{C} / \mathrm{m}^{2}\right)$ & $a$ & $0.01(\mathrm{MV} / \mathrm{m})$ \\
$P_{I}$ & $0.239\left(\mathrm{C} / \mathrm{m}^{2}\right)$ & $q$ & $500.0(\mathrm{MV} / \mathrm{m})^{2}$ \\
$P_{90 I}$ & $0.015\left(\mathrm{C} / \mathrm{m}^{2}\right)$ & $Y$ & $20000(\mathrm{MPa})$ \\
$\eta$ & 20.0 & $\eta_{2}$ & 20.0 \\
\hline
\end{tabular}

Table 3: Parameters for the 1-D piecewise ferroelastic switching model.

underprediction of the strains in the $E-\varepsilon$ curve. Simultaneously optimizing both the $\sigma-\varepsilon$ and $E-\varepsilon$ relations may be possible by using higher-order terms in the electromechanical coupling energy given by $(2)$.

The PLZT data in [2] illustrates the stress-dependence of the remanence and saturation polarization. For applied compressive stresses greater than $15 \mathrm{MPa}$, the remanence and saturation polarization significantly decrease. Since the remanence polarization is a fixed model parameter, the present formulation of the ferroelastic model should be limited to moderate stress regimes.

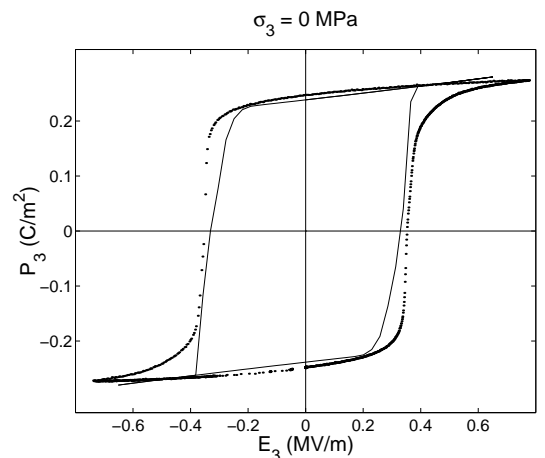

(a)

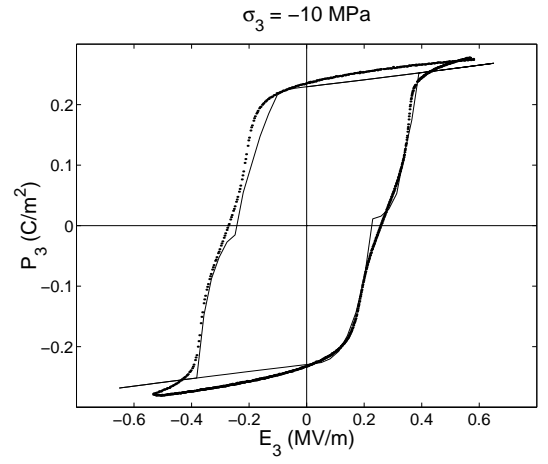

(c)

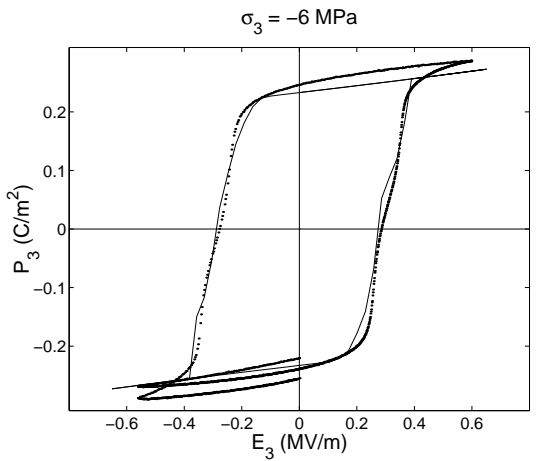

(b)

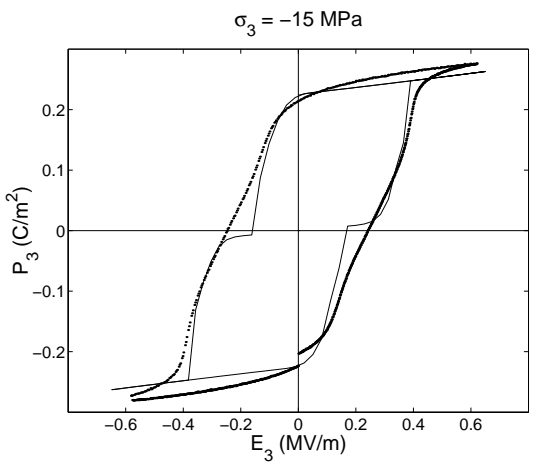

(d)

Figure 3: Macroscopic $E-P$ relation for varying applied stresses. Homogenized 1-D ferroelastic model $(-)$ and experimental PLZT data (..): (a) $\sigma_{3}=0 \mathrm{MPa}$, (b) $\sigma_{3}=-6 \mathrm{MPa}$, (c) $\sigma_{3}=-10 \mathrm{MPa}$, and (d) $\sigma_{3}=-15 \mathrm{MPa}$. 


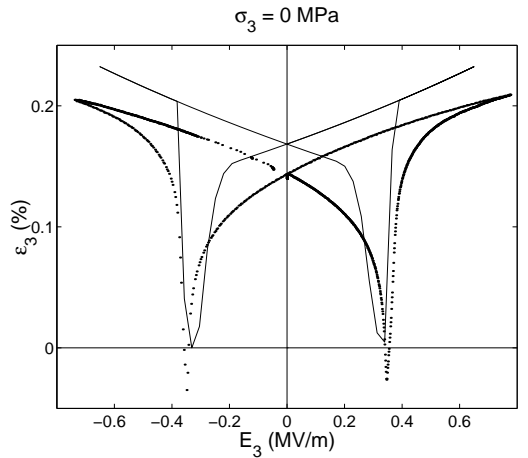

(a)

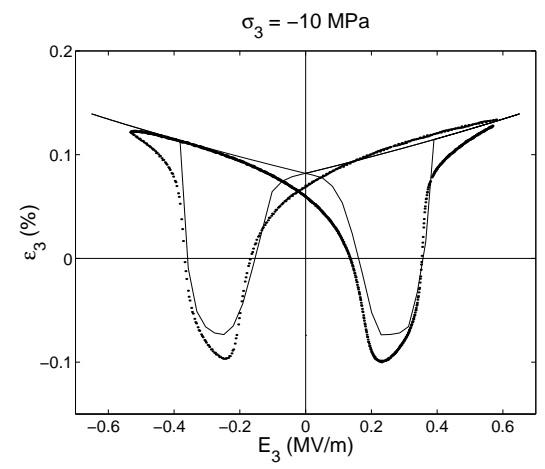

(c)

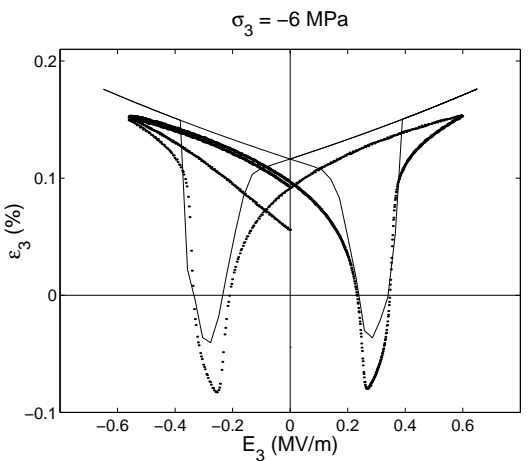

(b)

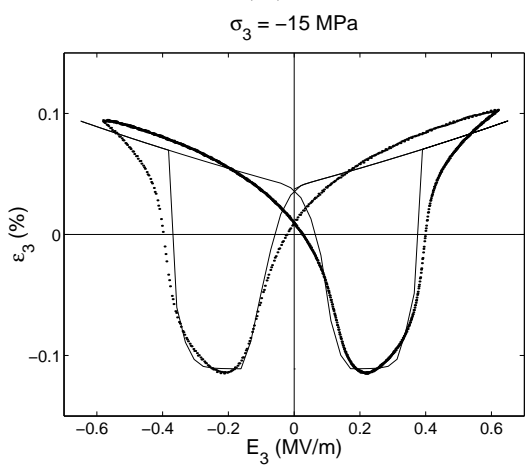

(d)

Figure 4: Macroscopic $E-\varepsilon$ relation for varying applied stresses. Homogenized 1-D ferroelastic model $(-)$ and experimental PLZT data ( ...): (a) $\sigma_{3}=0 \mathrm{MPa}$, (b) $\sigma_{3}=-6 \mathrm{MPa}$, (c) $\sigma_{3}=-10 \mathrm{MPa}$, and (d) $\sigma_{3}=-15 \mathrm{MPa}$.

\section{Concluding Remarks}

This paper summaries a model characterizing stress-induced $90^{\circ}$ and $180^{\circ}$ switching inherent to lead zirconate-titanate in a manner suitable for subsequent transducer and control design. Helmholtz and Gibbs energy functionals were used to characterize the electromechanical behavior of homogeneous, single crystal compounds. The construction is similar to that employed for SMA $[3,5,6,7]$ in the sense that it is a 1-D potential with three wells corresponding to the $\pm 180^{\circ}$ and $90^{\circ}$ equilibria. The construction of this functional is phenomenological but the resulting decrease in dimension significantly diminishes implementation time. The functionals are directly minimized to provide kernels for characterization in the absence of thermal relaxation or balanced with the relative thermal energy through Boltzmann principles to incorporate relaxation phenomena. The effects of material nonhomogeneities and polycrystallinity are incorporated by assuming that properties such as local coercive and interaction fields are manifestations of underlying distributions rather than constants. Stochastic homogenization in this manner provides a low order macroscopic model suitable for transducer and control design.

\section{Acknowledgements}

The research of BLB and RCS was supported through the NSF grant CMS-0099764 and the Air Force Office of Scientific Research under the grants AFOSR-F49620-01-1-0107 and AFOSR-FA955004-1-0203. The research of SS and SK was partially supported by the National Science Foundation through grant DMII 0134464. 


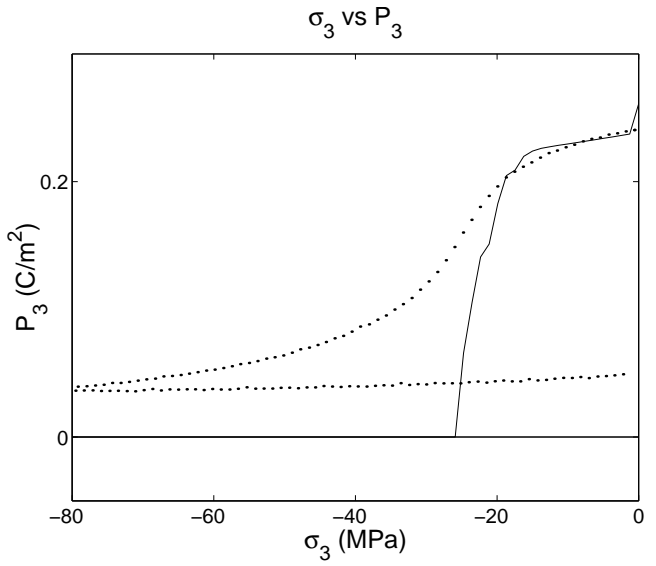

(a)

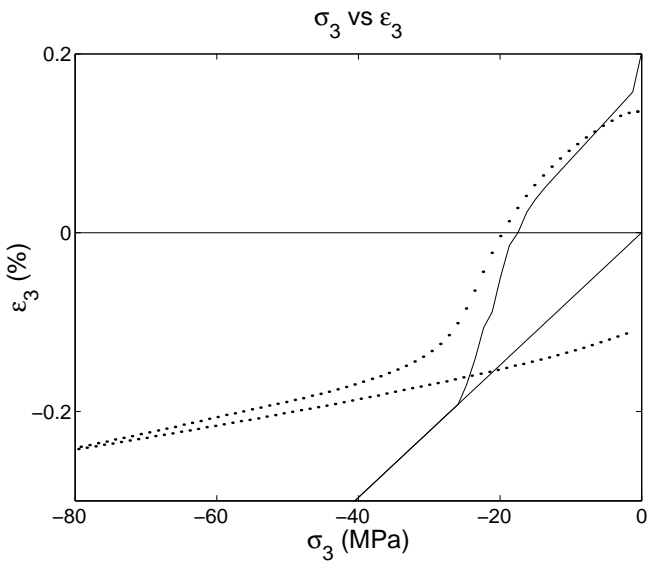

(b)

Figure 5: (a) Macroscopic $\sigma-P$ relation and (b) macroscopic $\sigma-\varepsilon$ relation. Homogenized 1-D ferroelastic model $(-)$ and experimental PLZT data $(\cdots)$.

\section{References}

[1] S.J. Kim, S. Seelecke, B.L. Ball, and R.C. Smith, "A Rate-Dependent Two-Dimensional Free Energy Model for Ferroelectric Single Crystals," Continuum Mechanics and Thermodynamics, submitted.

[2] C.S. Lynch, "The effect of uniaxial stress on the electro-mechanical response of 8/65/35 PLZT," Acta Materialia, 44(10), pp. 4137-4148, 1996.

[3] J.E. Massad and R.C. Smith, "A homogenized free energy model for hysteresis in thin-film shape memory alloys," CRSC Technical Report CRSC-TR04-26; International Journal on the Science and Technology of Condensed Matter Films, to appear.

[4] S. Nambu and D. A. Sagala, "Domain formation and elastic long-range interaction in ferroelectric perovskites," Physical Review B, 50(9), pp. 5838-5847, 1994.

[5] S. Seelecke and I. Müller, "Shape memory alloy actuators in smart structures — Modeling and simulation," ASME Applied Mechanics Reviews, 57(1), pp. 23-46, 2004.

[6] R.C. Smith, Smart Material Systems: Model Development, SIAM, Philadelophi, PA, 2005.

[7] R.C. Smith, S. Seelecke, M.J. Dapino and Z. Ounaies, "A unified model for hysteresis in ferroic materials," Proceedings of the SPIE, Volume 5049, pp. 88-99, Smart Structures and Materials, San Diego, CA, 2003.

[8] R.C. Smith, S. Seelecke, Z. Ounaies and J. Smith, "A free energy model for hysteresis in ferroelectric materials," Journal of Intelligent Material Systems and Structures, 14(11), pp. 719-739, 2003. 